BULL. AUSTRAL. MATH. SOC.

\title{
ON HERING'S FLAG TRANSITIVE PLANE \\ OF ORDER 27
}

\author{
M. L. narayana Rao, K. Kuppuswamy Rao \\ and $K$. SaTYANARAYANa
}

The full collineation group of the flag transitive plane of order

27 constructed by Hering is determined. It is shown that the stabilizer of the origin of this plane is of order 2184 .

1.

A collineation group $G$ of an affine plane $\pi$ is defined to be flag transitive on $\pi$ if $G$ is transitive on incident point line pairs or flags of $\pi$. The aim of this paper is to find the full collineation group of the flag transitive plane of order 27 constructed by Hering [2]. Recently the first two authors [4] in a joint paper discussed some properties of Hering's plane $\pi_{H}$.

2.

We give a brief description of Hering's plane. Let 0 be a $3 \times 3$ zero matrix over $G F(3)$ and let

$$
s=\left(\begin{array}{cc}
A_{1} & 0 \\
0 & A_{2}
\end{array}\right), \quad r=\left(\begin{array}{ll}
B_{1} & B_{2} \\
B_{3} & B_{4}
\end{array}\right), \quad h=\left(\begin{array}{cc}
0 & C_{1} \\
C_{2} & 0
\end{array}\right) \text {, }
$$

where

Received 10 August 1981. Research supported by UGC Grant F.23-1176/79 (SRII/SRIII). 
TABLE 2.1

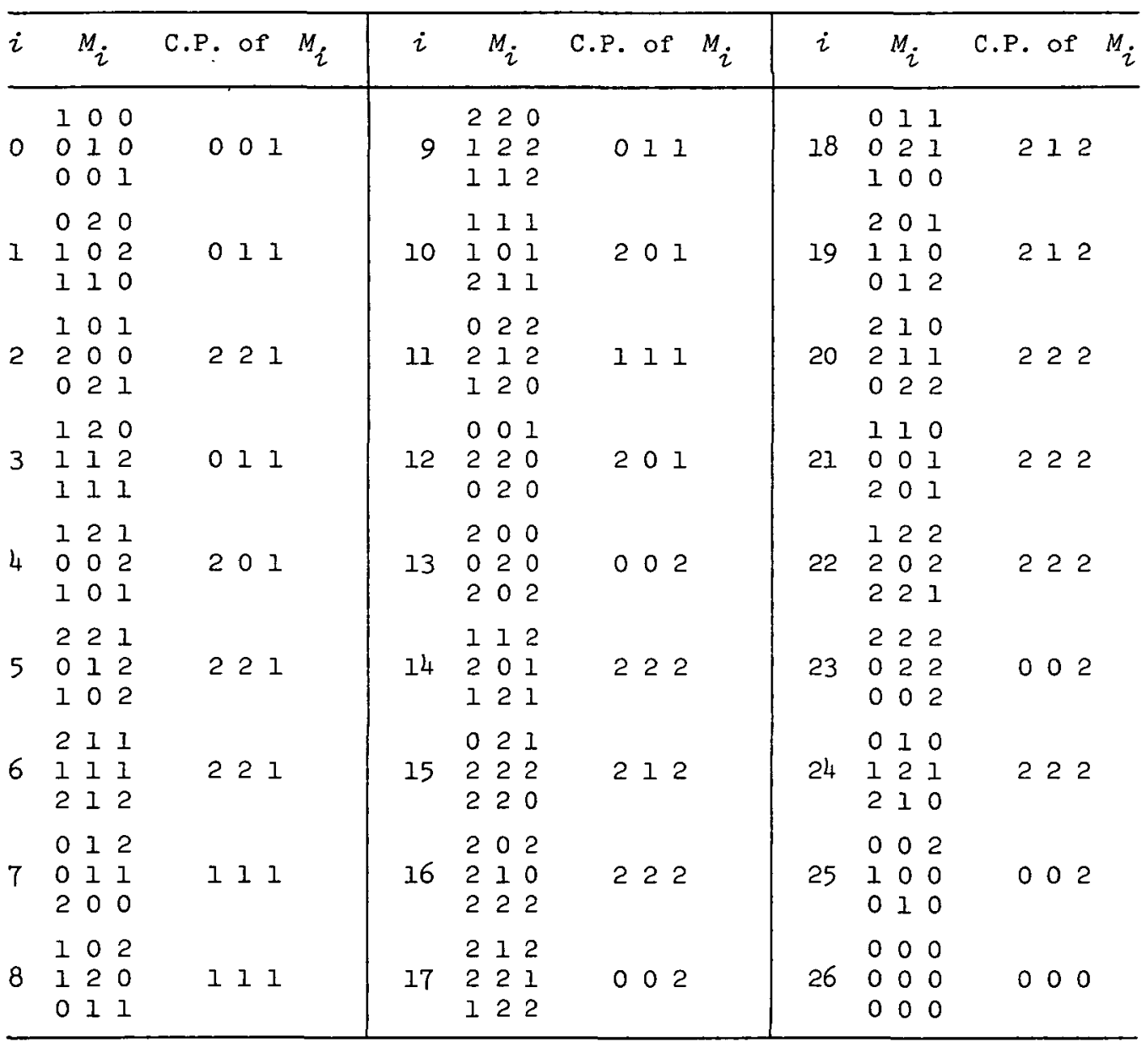

The entry $a b c$ under the heading C.P. of $M_{i}$ means that the matrix $M_{i}$ has the characteristic polynomial $-\lambda^{3}+a \lambda^{2}+b \lambda+c$. 


$$
\begin{aligned}
& A_{1}=\left(\begin{array}{lll}
0 & 2 & 0 \\
2 & 0 & 2 \\
1 & 0 & 0
\end{array}\right), \quad A_{2}=\left(\begin{array}{lll}
2 & 0 & 1 \\
2 & 0 & 0 \\
0 & 2 & 0
\end{array}\right), B_{1}=\left(\begin{array}{lll}
2 & 0 & 0 \\
2 & 1 & 0 \\
1 & 2 & 2
\end{array}\right), B_{2}=\left(\begin{array}{lll}
2 & 0 & 0 \\
2 & 1 & 0 \\
1 & 2 & 2
\end{array}\right), \\
& B_{3}=\left(\begin{array}{lll}
2 & 0 & 0 \\
2 & 1 & 0 \\
2 & 2 & 2
\end{array}\right), \quad B_{4}=\left(\begin{array}{lll}
1 & 0 & 0 \\
1 & 2 & 0 \\
2 & 1 & 1
\end{array}\right), C_{1}=\left(\begin{array}{lll}
2 & 0 & 0 \\
2 & 2 & 0 \\
0 & 1 & 2
\end{array}\right), C_{2}=\left(\begin{array}{lll}
1 & 0 & 0 \\
1 & 1 & 0 \\
2 & 2 & 1
\end{array}\right) \text {. }
\end{aligned}
$$

All the matrices in this paper are over $\mathrm{GF}(3)$. Let $L_{26}$ and $L_{27}$ be three dimensional subspaces of $V(6,3)$ defined by basis vectors as

$$
\begin{aligned}
& L_{26}=\langle(1,0,0,0,0,0),(0,1,0,0,0,0),(0,0,1,0,0,0)\rangle, \\
& L_{27}=\langle(0,0,0,1,0,0),(0,0,0,0,1,0),(0,0,0,0,0,1)\rangle .
\end{aligned}
$$

Let $L_{i}=L_{26^{r s}} s^{i}, 0 \leq i \leq 12, L_{13+i}=L_{27^{r s}}{ }^{i}, 0 \leq i \leq 12$. The incidence structure $\pi_{H}$ with $L_{i} \quad(0 \leq i \leq 27)$ and their cosets in the additive group of $V(6,3)$ as lines and vectors of $V(6,3)$ as points with inclusion as incidence relation is the flag transitive plane of order 27 constructed by Hering [2]. The subspace representing the lines $L_{i}$ may also be realised by the following procedure.

Let

$$
\begin{gathered}
M_{0}=\left(\begin{array}{lll}
1 & 0 & 0 \\
0 & 1 & 0 \\
0 & 0 & 1
\end{array}\right), M_{i+1}=A_{1}^{-1} M_{i 2} A_{1} \quad(0 \leq i \leq 11), M_{13}=\left(\begin{array}{lll}
2 & 0 & 0 \\
0 & 2 & 0 \\
2 & 0 & 2
\end{array}\right), \\
M_{13+i}=A_{1}^{-1} M_{13+i-1} A_{2} \quad(1 \leq i \leq 12), M_{26}=\left(\begin{array}{lll}
0 & 0 & 0 \\
0 & 0 & 0 \\
0 & 0 & 0
\end{array}\right) .
\end{gathered}
$$

Let

$$
z_{i}=\left\{(x, y, z, p, q, r) \mid x, y, z \in \mathrm{GF}(3),(p, q, r)=(x, y, z) M_{i}\right\}
$$

for $0 \leq i \leq 26$ and $z_{27}=L_{27}$. It is easily verified that $z_{i}=L_{i}$ for $0 \leq i \leq 27$. The set of matrices $M_{i}$ is given in Table 2.1.

3.

Any nonsingular linear transformation $T$ of $V(6,3)$ which permutes the subspaces $L_{i}, 0 \leq i \leq 27$, among themselves induces a collineation 
of $\pi_{H}$ fixing the point corresponding to the zero vector [1, Satz 19], [3, p. 208]. We may denote the collineation by $T$ itself. We now determine the group $G_{0}$ of all collineations which fix the point corresponding to the zero vector. Obviously the linear transformations $s, r$ and $h$ given earlier are elements of $G_{0}$. The actions of $s, r$ and $h$ restricted to the Iines $L_{i}, 0 \leq i \leq 27$, are given below:

$s:(26)(27)(0,1, \ldots, 12)(13,14, \ldots, 25)$;

$r:(0,26)(13,27)(1,12)(2,19)(3,4)(5,18)(6,15)(7,24)(8,21)$ $(9,10)(11,20)(14,25)(16,17)(22,23)$;

$h:(26,27)(0,13)(1,17,3,25,9,23)(2,21,6,24,5,20)$

$$
(4,16,12,22,10,14)(7,15,8,19,11,18) \text {. }
$$

Hence $(\ldots, a, b, c, \ldots)$ means $L_{a}$ is mapped onto $L_{b}$ and so on.

THEOREM 3.1. Any collineation of $\pi_{H}$ which fixes $L_{26}$ also fixes $L_{27}$

Proof. [4, Theorem 5.1, p. 343].

THEOREM 3.2. There is no collineation which fixes 26 and 27 and maps 0 onto 13 .

Proof. Here and in the course of this paper line $a$ means $L_{a}$. Any collineation which fixes the lines 26 and 27 and sends 0 onto 13 is of the form $\left(\begin{array}{ll}A & 0 \\ 0 & B\end{array}\right)$ where $A$ and $B$ are $3 \times 3$ nonsingular matrices over $G F(3)$ and 0 is the zero matrix and $B=A\left(\begin{array}{lll}2 & 0 & 0 \\ 0 & 2 & 0 \\ 2 & 0 & 2\end{array}\right)$. A further necessary and sufficient condition on $A$ is for each $i, 0 \leq i \leq 26$, $A^{-1} M_{i} A=M_{j}\left(\begin{array}{lll}2 & 0 & 0 \\ 0 & 2 & 0 \\ 1 & 0 & 2\end{array}\right)$ for some $j, 0 \leq j \leq 26$. But $M_{7}\left(\begin{array}{lll}2 & 0 & 0 \\ 0 & 2 & 0 \\ 1 & 0 & 2\end{array}\right)=\left(\begin{array}{lll}2 & 2 & 1 \\ 1 & 2 & 2 \\ 1 & 0 & 0\end{array}\right)$ has the characteristic polynomial $-\lambda^{3}+\lambda^{2}+2 \lambda+2$ which is not the characteristic polynomial of any $M_{i}, 0 \leq i \leq 26$. Hence there is no collineation that fixes lines 26 and 27 and sends line 0 to line 13. We may conclude that the sets of lines $\{0,1, \ldots, 12\}$ and $\{13,14, \ldots, 25\}$ are invariant under the group of all collineations 
fixing lines 26 and 27 .

THEOREM 3.3. The group of all collineations fixing lines $26,27,0$ and 13 is generated by $h^{2}$.

Proof. Any nonsingular linear transformation which fixes the lines $26,27,0$ and 13 is of the form $\left(\begin{array}{ll}A & 0 \\ 0 & A\end{array}\right)$ where $A$ is a nonsingular $3 \times 3$ matrix over $\mathrm{GF}(3)$ and 0 is the zero matrix and further $A M_{13}=M_{13} A$. Then $A$ is of the form $\left(\begin{array}{lll}a & 0 & 0 \\ x & y & 0 \\ p & q & a\end{array}\right)$ with $a \neq 0 \neq y$. On examination of Table 2.1 we find that $M_{1}, M_{3}$ and $M_{9}$ are the only matrices having the characteristic polynomial $-\lambda^{3}+\lambda+1$ and in fact, they are similar. Thus if the above linear transformation is a collineation, then it must map 1,3 and 9 among themselves. This forces $A$ to be an element of the group generated by the matrix $\left(\begin{array}{lll}2 & 0 & 0 \\ 1 & 2 & 0 \\ 2 & 2 & 2\end{array}\right)$ which forces in its turn that any collineation that fixes the lines 26 , 27,0 and 13 is a power of $h^{2}$ and the group of all such collineations is of order 6 .

THEOREM 3.4. The group $G_{0}$ of all collineations which fix the point corresponding to the zero vector and permute the lines $L_{i}$ among themselves is generated by $\{s, r, h\}$.

Proof. Let $H_{26}$ be the group of collineations from $G_{0}$ which fix 26 . Since the restriction of $G_{0}$ to the lines $L_{i}$ is transitive on the set of lines $L_{i}, 0 \leq i \leq 27, G_{0}$ is given by a disjoint union of cosets of $H_{26}$ by the expression

$$
\bigcup_{i=0}^{i=27} H_{26^{a}}{ }_{i}
$$

where for each $i, a_{i}$ is a collineation from $G_{0}$ which sends $L_{26}$ onto $L_{i}$. We now determine $H_{26}$. The group $H_{26}$ contains the collineation $s$ which is transitive on the set of lines $\{0,1, \ldots, 12\}$. In view of 
Theorem 3.2 we may conclude that $H_{26}$ is transitive on the set of lines $\{0,1,2, \ldots, 12\}$. Let $K_{26,0}$ be the group of all collineations from $H_{26}$ that fix line 0 . Since the collineation $s^{i}$ fixes the line 26 and sends line 0 to line $i$ for $0 \leq i \leq 12$ we may express $H_{26}$ as a disjoint union of cosets of $K_{26,0}$ given by the expression

$$
H_{26}=\bigcup_{i=0}^{i=12} K_{26,0^{s}} \text {. }
$$

We have to determine $K_{26,0}$. Suppose a collineation fixes the lines 26 and zero. In view of Theorem 3.1 we may say that it fixes the line 27 also. Further its conjugate by $r$ fixes lines 0 and 13 showing that whenever line 0 is fixed by a collineation, the line 13 also is fixed by the same collineation. Thus if a collineation fixes lines 26 and 0 , then it fixes lines 27 and 13 as well. The group $K_{26,0}$ consists of all collineations from $H_{26}$ which fix simultaneously lines $26,27,0$ and 13. Theorem 3.3 implies that $K_{26,0}=\left\langle h^{2}\right\rangle$. Then

$$
H_{26}=\bigcup_{i=0}^{i=12}\left\langle h^{2}\right\rangle s^{i} \text {. }
$$

Let $a_{26}$ be the identity, $a_{i}=r s^{i}, 0 \leq i \leq 12$, and $a_{13+i}=h r s^{i}$, $0 \leq i \leq 12$, and $a_{27}=h$. It is easily seen that $a_{i}$ sends line 26 onto line $i$ for $0 \leq i \leq 27$. We may now conclude that

$$
G_{0}=\bigcup_{j=0}^{27} \bigcup_{i=0}^{12}\left\langle h^{2}\right\rangle s^{i} a_{j}
$$

and $G_{0}$ contains $6 \cdot 13 \cdot 28=2184$ elements. If $G^{\prime}$ is the group of all translations, then the full collineation group $G$ of $\pi_{H}$ is given by $G=\left\langle G^{\prime}, G_{0}\right\rangle$. 


\section{References}

[1] Johannes André, "Über nicht-Desarguessche Ebenen mit transitiver Translationsgruppe", Math. Z. 60 (1954), 156-186.

[2] Christoph Hering, "Eine nicht-desarguessche zweifach transitive affine Ebene der Ordnung 27 ", Abh. Math. Sem. Univ. Hamburg 34 (1970), 203-208.

[3] Günter Pickert, Projecktive Ebenen (Die Grundlehren der mathematischen Wissenschaften, 80. Springer-Verlag, Berlin, Gottingen, Heidelberg, 1955).

[4] M.L. Narayana Rao and K. Kuppuswamy Rao, "A new flag transitive affine plane of order 27 ", Proc. Amer. Math. Soc. 59 (1976), 337-345.

Department of Mathematics,

University College of Science,

Osmania University,

Hyderabad 500007 ,

India.

Present address for Dr K. Kuppuswamy Rao:

Department of Mathematics,

Rivers State College of Education,

PMB 5047,

Port Harcourt,

Nigeria. 